\title{
Gravitational form factors of a spin one particle
}

\author{
Maxim V. Polyakov ${ }^{1,2}$ and Bao-Dong Sun ${ }^{3,4, *}$ \\ ${ }^{1}$ Petersburg Nuclear Physics Institute, Gatchina, 188300 St. Petersburg, Russia \\ ${ }^{2}$ Institut für Theoretische Physik II, Ruhr-Universität Bochum, D-44780 Bochum, Germany \\ ${ }^{3}$ Institute of High Energy Physics, Chinese Academy of Sciences, \\ Beijing 100049, People's Republic of China \\ ${ }^{4}$ School of Physics, University of Chinese Academy of Sciences, \\ Beijing 100049, People's Republic of China
}

(Received 10 April 2019; published 12 August 2019)

\begin{abstract}
We define the form factors of the quark and gluon symmetric energy-momentum tensor (EMT). The static EMT is related to the spatial distributions of energy, spin, pressure, and shear forces. They are obtained in the form of a multipole expansion. The relations between gravitational form factors and the generalized parton distributions are given.
\end{abstract}

DOI: 10.1103/PhysRevD.100.036003

\section{INTRODUCTION}

The gravitational form factors (GFFs) contain the information of the spatial distributions of energy, spin, pressure, and shear forces inside the system [1]. The GFFs are defined through the matrix elements of the symmetric energy-momentum tensor (EMT). More details can be found in the recent papers [2,3]. For spin one particles, the GFFs, or EMT FFs, have been discussed in the literature [4-6], but, to our best knowledge, EMT-nonconserving FFs are either not discussed [4,5] or incomplete [6]. Thus we introduced a definition for individual quark and gluon EMT FFs for spin one particles in Sec. II. In the Breit frame, we find that matrix elements of EMT can be expressed in terms of the multipole expansion for energy density, pressure, and shear forces distributions; see Sec. III. By considering the Mellin moments of the vector generalized parton distributions (GPDs), the sum rules between the GPDs and EMT FFs are found in Sec. IV.

The EMT of QCD can be obtained by varying the action $S_{\text {grav }}$ of QCD coupled to a weak classical torsionless gravitational background field with respect to the metric $g^{\mu \nu}(x)$ of this curved background field according to $[2,7]$

$$
\hat{T}_{\mu \nu}(x)=\frac{2}{\sqrt{-g}} \frac{\delta S_{\text {grav }}}{\delta g^{\mu \nu}(x)},
$$

\footnotetext{
*sunbd@ihep.ac.cn
}

Published by the American Physical Society under the terms of the Creative Commons Attribution 4.0 International license. Further distribution of this work must maintain attribution to the author(s) and the published article's title, journal citation, and DOI. Funded by SCOAP ${ }^{3}$. where $g$ denotes the determinant of the metric (the signature of the metric we use is +---$)$. This procedure yields a symmetric Belinfante-Rosenfeld EMT. The quark and gluon contributions to the total EMT operator are given by

$$
\begin{aligned}
& T_{q}^{\mu \nu}=\frac{1}{4}\left[\bar{\psi}_{q}\left(-i \overleftarrow{\mathcal{D}}^{\mu} \gamma^{\nu}-i \overleftarrow{\mathcal{D}}^{\nu} \gamma^{\mu}+i \overrightarrow{\mathcal{D}}^{\mu} \gamma^{\nu}+i \overrightarrow{\mathcal{D}}^{\nu} \gamma^{\mu}\right) \psi_{q}\right. \\
& \left.-g^{\mu \nu} \bar{\psi}_{q}\left(-\frac{i}{2} \overleftarrow{D}+\frac{i}{2} \vec{D}-m_{q}\right) \psi_{q}\right] \text {, } \\
& T_{g}^{\mu \nu}=F^{a, \mu \eta} F^{a,{ }_{\eta}{ }^{\nu}+\frac{1}{4}} g^{\mu \nu} F^{a, \kappa \eta} F^{a,}{ }{ } \eta .
\end{aligned}
$$

Here $\overrightarrow{\mathcal{D}}_{\mu}=\vec{\partial}_{\mu}+i g t^{a} A_{\mu}^{a}$ and $\overleftarrow{\mathcal{D}}_{\mu}=\overleftarrow{\partial}_{\mu}-i g t^{a} A_{\mu}^{a}$ with arrows indicating which fields are differentiated, $F_{\mu \nu}^{a}=$ $\partial_{\mu} A_{\nu}^{a}-\partial_{\nu} A_{\mu}^{a}-g f^{a b c} A_{\mu}^{b} A_{\nu}^{c}$, and the SU(3) color group generators satisfy the algebra $\left[t^{a}, t^{b}\right]=i f^{a b c} t^{c}$ and are normalized as $\operatorname{tr}\left(t^{a} t^{b}\right)=\frac{1}{2} \delta^{a b}$. The total EMT is conserved

$$
\partial^{\mu} \hat{T}_{\mu \nu}=0, \quad \hat{T}_{\mu \nu}=\sum_{q} \hat{T}_{\mu \nu}^{q}+\hat{T}_{\mu \nu}^{g}
$$

\section{DEFINITION OF EMT FORM FACTORS}

We use the covariant normalization $\left\langle p^{\prime}, \sigma^{\prime} \mid p, \sigma\right\rangle=$ $2 p^{0}(2 \pi)^{3} \delta^{(3)}\left(\boldsymbol{p}^{\prime}-\boldsymbol{p}\right) \delta_{\sigma \sigma^{\prime}}$ of one-particle states and introduce the kinematic variables $P=\frac{1}{2}\left(p^{\prime}+p\right)$ and $\Delta=$ $p^{\prime}-p, t=\Delta^{2}$. The EMT form factors of a spin one particle in QCD we define as ${ }^{1}$

\footnotetext{
${ }^{1}$ We chose the naming of the form factors in line with the naming used in Refs. [1,2] for spin- 0 and spin- $1 / 2$ particles.
} 
TABLE I. The notations of EMT FFs in the literature (the FFs in $[9,10]$ are not exactly EMT FFs) and their values in free theory obtained by the Proca Lagrangian. In Ref. [4] there is a sign mistake in the term corresponding to our $\left(\epsilon_{\mu} \Delta_{\nu}+\epsilon_{\nu} \Delta_{\mu}\right) \epsilon^{\prime *} \cdot P$ in $E^{a}(t)$ 's coefficient. In Ref. [6], the authors missed one term that should correspond to the $\bar{c}_{1}^{a}$ term here. The result of Ref. [11], which appeared during the preparation of this paper, coincides with our result.

\begin{tabular}{lccccccccc}
\hline \hline This work & $A_{0}$ & $A_{1}$ & $D_{0}$ & $D_{1}$ & $J$ & $E$ & $\bar{f}$ & $\bar{c}_{0}$ & $\bar{c}_{1}$ \\
\hline Free theory & 1 & 0 & $1+4 h$ & 0 & 1 & 1 & 0 & 0 & 0 \\
Holstein [4] & $F_{1}$ & $4 F_{5}$ & $-2 F_{2}$ & $8 F_{6}$ & $F_{3}$ & $-2 F_{4}$ & $\ldots$ & $\ldots$ & $\ldots$ \\
Abidin and Carlson [5] & $A$ & $-2 E$ & $C$ & $-8 F$ & $A+B$ & $D$ & $\ldots$ & $\ldots$ & $\ldots$ \\
Taneja et al. [6] & $\mathcal{G}_{1}$ & $-2 \mathcal{G}_{2}$ & $-\mathcal{G}_{3}$ & $-2 \mathcal{G}_{4}$ & $\frac{1}{2} \mathcal{G}_{5}$ & $-\frac{1}{2} \mathcal{G}_{6}$ & $\frac{1}{2 m^{2}} \mathcal{G}_{7}$ & $\frac{1}{4 m^{2}} \mathcal{G}_{7}+\mathcal{G}_{8}$ & $\ldots$ \\
Cosyn et al. [11] & $\mathcal{G}_{1}$ & $-2 \mathcal{G}_{2}$ & $-\mathcal{G}_{3}$ & $-2 \mathcal{G}_{4}$ & $\frac{1}{2} \mathcal{G}_{5}$ & $-\frac{1}{2} \mathcal{G}_{6}$ & $\frac{1}{2} \mathcal{G}_{7}$ & $\frac{1}{4} \mathcal{G}_{7}+\mathcal{G}_{8}$ & $-2 \mathcal{G}_{9}$ \\
Cosyn et al. [9] generalized form factors & $A_{2,0}^{a}$ & $-2 C_{2,0}^{a}$ & $-4 F_{2}^{a}$ & $-8 G_{2}^{a}$ & $\frac{1}{2} B_{2,0}^{a}$ & $D_{2,1}^{a}$ & $\sim E_{2,0}^{a}$ & $\ldots$ & $\ldots$ \\
Hoodbhoy et al. [10] reduced matrix elements & $a_{2}-\frac{1}{3} d_{2}$ & $\cdots$ & $\ldots$ & $\ldots$ & $\ldots$ & $\ldots$ & $\sim d_{2}$ & $\ldots$ & $\ldots$ \\
\hline \hline
\end{tabular}

$$
\begin{aligned}
\left\langle p^{\prime}, \sigma^{\prime}\left|\hat{T}_{\mu \nu}^{a}(x)\right| p, \sigma\right\rangle= & {\left[2 P_{\mu} P_{\nu}\left(-\epsilon^{\prime *} \cdot \epsilon A_{0}^{a}(t)+\frac{\epsilon^{\prime *} \cdot P \epsilon \cdot P}{m^{2}} A_{1}^{a}(t)\right)+2\left[P_{\mu}\left(\epsilon_{\nu}^{\prime *} \epsilon \cdot P+\epsilon_{\nu} \epsilon^{\prime *} \cdot P\right)+P_{\nu}\left(\epsilon_{\mu}^{\prime *} \epsilon \cdot P+\epsilon_{\mu} \epsilon^{\prime *} \cdot P\right)\right] J^{a}(t)\right.} \\
& +\frac{1}{2}\left(\Delta_{\mu} \Delta_{\nu}-g_{\mu \nu} \Delta^{2}\right)\left(\epsilon^{\prime *} \cdot \epsilon D_{0}^{a}(t)+\frac{\epsilon^{\prime *} \cdot P \epsilon \cdot P}{m^{2}} D_{1}^{a}(t)\right) \\
& +\left[\frac{1}{2}\left(\epsilon_{\mu} \epsilon_{\nu}^{\prime *}+\epsilon_{\mu}^{\prime *} \epsilon_{\nu}\right) \Delta^{2}-\left(\epsilon_{\mu}^{\prime *} \Delta_{\nu}+\epsilon_{\nu}^{\prime *} \Delta_{\mu}\right) \epsilon \cdot P+\left(\epsilon_{\mu} \Delta_{\nu}+\epsilon_{\nu} \Delta_{\mu}\right) \epsilon^{\prime *} \cdot P-4 g_{\mu \nu} \epsilon^{\prime *} \cdot P \epsilon \cdot P\right] E^{a}(t) \\
& \left.+\left(\epsilon_{\mu} \epsilon_{\nu}^{\prime *}+\epsilon_{\mu}^{\prime *} \epsilon_{\nu}-\frac{\epsilon^{\prime *} \cdot \epsilon}{2} g_{\mu \nu}\right) m^{2} \bar{f}^{a}(t)+g_{\mu \nu}\left(\epsilon^{\prime *} \cdot \epsilon m^{2} \bar{c}_{0}^{a}(t)+\epsilon^{\prime *} \cdot P \epsilon \cdot P \bar{c}_{1}^{a}(t)\right)\right] e^{i\left(p^{\prime}-p\right) x}
\end{aligned}
$$

where $a=g, u, d, \ldots$, and the polarization vectors $\epsilon_{\mu}^{\prime}=\epsilon_{\mu}\left(p^{\prime}, \sigma^{\prime}\right), \epsilon_{\mu}=\epsilon_{\mu}(p, \sigma)$, and $\sigma= \pm 1,0$. There are nine GFFs for each quark flavor or gluon for a spin one particle. The six quark and gluon form factors (FFs) $A_{0,1}^{a}$, $D_{0,1}^{a}, J^{a}$, and $E^{a}(t)$ are individually EMT conserving, and the other three FFs, $\bar{f}^{a}$ and $\bar{c}_{0,1}^{a}(t)$, are not. ${ }^{2}$ As discussed in Ref. [2], all individual quark and gluon FFs depend on the renormalization scale, which we do not indicate for brevity. Because of EMT conservation, Eq. (3), the constraints $\sum_{a} \bar{f}^{a}(t)=0$ and $\sum_{a} \bar{c}_{0,1}^{a}(t)=0$ hold, and the total form factors $A_{0,1}(t), D_{0,1}(t), J(t)$, and $E(t)$ are renormalization scale invariant where we defined $A_{0}(t) \equiv \sum_{a} A_{0}^{a}(t)$ and analogously for other form factors. Some of the notations for EMT FFs in the literature are listed in Table I. The generalized form factors in [9] and the reduced matrix elements in [10] are connected with the gravitational form factors as shown in Table I.

\section{A. EMT form factors in free field theory}

In the free field theory, the massive spin one particles are described by the Proca Lagrangian,

$$
\mathcal{L}=-\frac{1}{4} U_{\mu \nu} U^{\mu \nu}+\frac{1}{2} m^{2} A_{\mu} A^{\mu},
$$

\footnotetext{
${ }^{2}$ For the particle with integer spin $J$ there are $(4 J+2)$ conserving and $(2 J+1)$ nonconserving EMT FFs [8].
}

where $A_{\mu}$ is a massive vector field and the field tensor is

$$
U_{\mu \nu}=\partial_{\mu} A_{\nu}-\partial_{\nu} A_{\mu}
$$

The EMT corresponding to the Proca Lagrangian is given by

$$
\hat{T}_{\mu \nu}^{\text {(Proca) }}=-U_{\mu \rho} U_{\nu}{ }^{\rho}-g_{\mu \nu} \mathcal{L}+m^{2} A_{\mu} A_{\nu} .
$$

The action $S_{\text {grav }}$ can be modified by adding a nonminimal term for interaction with the gravity:

$$
S_{\text {grav }}=\int d^{4} x \sqrt{-g}\left(-\frac{1}{4} U_{\mu \nu} U^{\mu \nu}+\frac{1}{2} m^{2} A_{\mu} A^{\mu}+\frac{1}{2} h R A^{2}\right) .
$$

Here, $R$ is the Riemann scalar. With this term added, the EMT in the free field theory becomes

$$
\begin{aligned}
\hat{T}_{\mu \nu}^{\text {improve }} & =\hat{T}_{\mu \nu}^{\text {(Proca) }}+\theta_{\mu \nu}^{\text {improve }}, \\
\text { with } \quad \theta_{\mu \nu}^{\text {improve }} & =-h\left(\partial_{\mu} \partial_{\nu}-g_{\mu \nu} \partial^{2}\right) A^{2} .
\end{aligned}
$$

The value of the parameter $h$ depends on the physics problem one is considering. With this improved EMT, one can obtain the free theory values of the total FFs [4] as shown in Table I. 


\section{THE STATIC EMT AND STRESS TENSOR}

Before discussing the components of EMT in Eq. (4), let us review the spin and quadrupole operators. For particles with spin $S \geq 1$, the quadrupole operator is the $(2 S+1) \times$ $(2 S+1)$ matrix:

$$
\hat{Q}^{i k}=\frac{1}{2}\left(\hat{S}^{i} \hat{S}^{k}+\hat{S}^{k} \hat{S}^{i}-\frac{2}{3} S(S+1) \delta^{i k}\right) \quad(i, j, k=1,2,3),
$$

which is expressed in terms of the spin operator $\hat{S}^{i}$. The spin operator can be expressed in terms of the SU(2) ClebschGordan coefficients (in the spherical basis):

$$
\hat{S}_{\sigma^{\prime} \sigma}^{\lambda}=\sqrt{S(S+1)} C_{S \sigma 1 \lambda}^{S \sigma^{\prime}}\left(\lambda=0, \pm 1 . \sigma, \sigma^{\prime}=0, \ldots, \pm J\right) .
$$

In the spin one case, it is equivalent to

$$
\hat{S}_{\sigma^{\prime} \sigma}^{i}=i \epsilon^{i j k} \hat{\epsilon}_{\sigma}^{* j} \hat{\epsilon}_{\sigma^{\prime}}^{k} \quad(i, j, k=1,2,3),
$$

where $\epsilon^{\mu}(0, \sigma)=\left(0, \hat{\epsilon}_{\sigma}\right)$ is the rest frame spin one polarization vectors,

$$
\hat{\epsilon}_{ \pm}=\mp \frac{1}{\sqrt{2}}(1, \pm i, 0), \quad \hat{\epsilon}_{0}=(0,0,1) .
$$

Applying the boost operator $L(p)$ from the rest frame $k^{\mu}=(m, 0,0,0)$ to any frame $p^{\mu}\left(=L_{\nu}^{\mu} k^{\nu}\right)$, one has $[12,13]$

$$
\epsilon^{\mu}(p, \sigma)=\left(\frac{\vec{p} \cdot \hat{\epsilon}_{\sigma}}{m}, \hat{\epsilon}_{\sigma}+\frac{\vec{p} \cdot \hat{\epsilon}_{\sigma}}{m(m+E)} \vec{p}\right)
$$

where $\sigma=\{+,-, 0\}$ and $m$ and $E=\sqrt{|\vec{p}|^{2}+m^{2}}$ are the rest mass and energy of the state.

In the Breit frame, the initial (final) momentum $p^{\mu}\left(p^{\prime \mu}\right)$ has the relation $P^{\mu}=\left(p^{\mu}+p^{\prime \mu}\right) / 2=(E, 0,0,0)$ and $\Delta^{\mu}=p^{\prime \mu}-p^{\mu}=(0, \vec{\Delta})$. So $\vec{p}=-\vec{p}^{\prime}=-\vec{\Delta} / 2$ and $p^{0}=$ $p^{\prime 0}=E=\sqrt{m^{2}-t / 4}$ with $t=\Delta^{2}$. In this frame, with the polarization vectors Eq. (15), Eq. (4) can be expressed as

$$
\begin{aligned}
\left\langle p^{\prime}, \sigma^{\prime}\left|\hat{T}_{a}^{00}(0)\right| p, \sigma\right\rangle= & 2 m^{2} \mathcal{E}_{0}^{a}(t) \delta_{\sigma^{\prime} \sigma}+\hat{Q}^{k l} \Delta^{k} \Delta^{l} \mathcal{E}_{2}^{a}(t), \\
\left\langle p^{\prime}, \sigma^{\prime}\left|\hat{T}_{a}^{0 j}(0)\right| p, \sigma\right\rangle= & i \epsilon^{j k l} \hat{S}_{\sigma^{\prime} \sigma}^{k} \Delta^{l} m \mathcal{J}^{a}(t), \\
\left\langle p^{\prime}, \sigma^{\prime}\left|\hat{T}_{a}^{i j}(0)\right| p, \sigma\right\rangle= & \frac{1}{2}\left(\Delta^{i} \Delta^{j}-\delta^{i j} \vec{\Delta}^{2}\right) \mathcal{D}_{0}^{a}(t) \delta_{\sigma^{\prime} \sigma}+\left(\Delta^{j} \Delta^{k} \hat{Q}^{i k}+\Delta^{i} \Delta^{k} \hat{Q}^{j k}-\vec{\Delta}^{2} \hat{Q}^{i j}-\delta^{i j} \Delta^{k} \Delta^{l} \hat{Q}^{k l}\right) \mathcal{D}_{2}^{a}(t) \\
& +\frac{1}{2 m^{2}}\left(\Delta^{i} \Delta^{j}-\delta^{i j} \vec{\Delta}^{2}\right) \Delta^{k} \Delta^{l} \hat{Q}^{k l} \mathcal{D}_{3}^{a}(t) \\
& +\left[\delta^{i j} \delta_{\sigma^{\prime} \sigma}\left(\frac{m^{2}}{6}+\frac{t}{12}\right)+\frac{1}{6} \Delta^{i} \Delta^{j} \delta_{\sigma^{\prime} \sigma}-2 m^{2} \hat{Q}^{i j}-\frac{m}{2(m+E)}\left(\Delta^{i} \Delta^{k} \hat{Q}^{k j}+\Delta^{j} \Delta^{k} \hat{Q}^{k i}\right)\right. \\
& \left.+\frac{1}{4}\left(\delta^{i j}-\frac{\Delta^{i} \Delta^{j}}{2(m+E)^{2}}\right) \Delta^{k} \Delta^{l} \hat{Q}^{k l}\right] \bar{f}^{a}(t) \\
& +\delta^{i j}\left\{\left[\delta_{\sigma^{\prime} \sigma}\left(\frac{t}{6}-m^{2}\right)+\frac{1}{2} \Delta^{k} \Delta^{l} \hat{Q}^{k l}\right] \bar{c}_{0}^{a}(t)+\frac{1}{4}\left(1-\frac{t}{4 m^{2}}\right)\left(\frac{t}{3} \delta_{\sigma^{\prime} \sigma}+\Delta^{k} \Delta^{l} \hat{Q}^{k l}\right) \bar{c}_{1}^{a}(t)\right\},
\end{aligned}
$$

where $\hat{Q}^{k l}=\left\langle S, \sigma^{\prime}\left|\hat{Q}^{k l}\right| S, \sigma\right\rangle$ are the matrix elements of the quadrupole operator and

$$
\begin{aligned}
\mathcal{E}_{0}^{a}(t)= & A_{0}^{a}(t)+\frac{1}{4} \bar{f}^{a}(t)-\frac{1}{2} \bar{c}_{0}^{a}(t)+\frac{t}{12 m^{2}}\left[-5 A_{0}^{a}(t)+3 D_{0}^{a}(t)+4 J^{a}(t)-2 E^{a}(t)+A_{1}^{a}(t)+\frac{1}{2} \bar{f}^{a}(t)+\bar{c}_{0}^{a}(t)+\frac{1}{2} \bar{c}_{1}^{a}(t)\right] \\
& -\frac{t^{2}}{24 m^{4}}\left[-A_{0}^{a}(t)+D_{0}^{a}(t)+2 J^{a}(t)-2 E^{a}(t)+A_{1}^{a}(t)+\frac{1}{2} D_{1}^{a}(t)+\frac{1}{4} \bar{c}_{1}^{a}(t)\right]+\frac{t^{3}}{192 m^{6}}\left[A_{1}^{a}(t)+D_{1}^{a}(t)\right] \\
\mathcal{E}_{2}^{a}(t)= & -A_{0}^{a}(t)+2 J^{a}(t)-E^{a}(t)+\frac{1}{2} A_{1}^{a}(t)+\frac{1}{4} \bar{f}^{a}(t)+\frac{1}{2} \bar{c}_{0}^{a}(t)+\frac{1}{4} \bar{c}_{1}^{a}(t) \\
& -\frac{t}{4 m^{2}}\left[-A_{0}^{a}(t)+D_{0}^{a}(t)+2 J^{a}(t)-2 E^{a}(t)+A_{1}^{a}(t)+\frac{1}{2} D_{1}^{a}(t)+\frac{1}{4} \bar{c}_{1}^{a}(t)\right]+\frac{t^{2}}{32 m^{4}}\left[A_{1}^{a}(t)+D_{1}^{a}(t)\right] \\
\mathcal{J}^{a}(t)= & J^{a}(t)+\frac{1}{2} \bar{f}^{a}(t)-\frac{t}{4 m^{2}}\left(J^{a}(t)-E^{a}(t)\right)
\end{aligned}
$$




$$
\begin{aligned}
& \mathcal{D}_{0}^{a}(t)=-D_{0}^{a}(t)+\frac{4}{3} E^{a}(t)+\frac{t}{12 m^{2}}\left[2 D_{0}^{a}(t)-2 E^{a}(t)+D_{1}^{a}(t)\right]-\frac{t^{2}}{48 m^{4}} D_{1}^{a}(t), \\
& \mathcal{D}_{2}^{a}(t)=-E^{a}(t), \\
& \mathcal{D}_{3}^{a}(t)=\frac{1}{4}\left[2 D_{0}^{a}(t)-2 E^{a}(t)+D_{1}^{a}(t)\right]-\frac{t}{16 m^{2}} D_{1}^{a}(t) .
\end{aligned}
$$

The details for obtaining Eqs. (16) and (17) are shown in the Appendix.

Because of the constraint $\sum_{a} \bar{f}^{a}(t)=0$ and $\sum_{a} \bar{c}_{0,1}^{a}(t)=0$, the total quark + gluon EMT drops $\bar{f}^{a}$ and $\bar{c}_{0,1}^{a}(t)$ terms, and so do $\mathcal{E}_{0,2}, \mathcal{J}$, and $\mathcal{D}_{0,2,3}(t)$. The free theory values of the total EMT FFs are listed in Table II. The $D$-term is defined as

$$
D \equiv \mathcal{D}_{0}(0)=\frac{1}{3}-4 h \text {. }
$$

Following Ref. [1], the static EMT $T^{\mu \nu}\left(\vec{r}, \sigma^{\prime}, \sigma\right)$ of the spin one particle is defined by Fourier transforming the EMT in Eqs. (16a)-(16c) with respect to $\vec{\Delta}$ as

$T_{a}^{\mu \nu}\left(\vec{r}, \sigma^{\prime}, \sigma\right)=\int \frac{d^{3} \Delta}{2 E(2 \pi)^{3}} e^{-i \vec{\Delta} \cdot \vec{r}}\left\langle p^{\prime}, \sigma^{\prime}\left|\hat{T}_{a}^{\mu \nu}(0)\right| p, \sigma\right\rangle$,

where $r=|\vec{r}|$.

\section{A. $T^{00}$ : Energy density}

Because of the presence of the EMT-nonconserving terms $\bar{f}^{a}$ and $\bar{c}_{0,1}^{a}$, the energy density $T^{00}\left(\vec{r}, \sigma^{\prime}, \sigma\right)$ can only be defined for the total system. The multipole expansion of the energy density is defined as [14]

$$
\begin{aligned}
T^{00}\left(\vec{r}, \sigma^{\prime}, \sigma\right) & =\int \frac{d^{3} \Delta}{2 E(2 \pi)^{3}} e^{-i \vec{\Delta} \cdot \vec{r}}\left\langle p^{\prime}, \sigma^{\prime}\left|\hat{T}^{00}(0)\right| p, \sigma\right\rangle \\
& =\varepsilon_{0}(r) \delta_{\sigma^{\prime} \sigma}+\varepsilon_{2}(r) \hat{Q}^{i j} Y_{2}^{i j}
\end{aligned}
$$

where

$$
\begin{array}{r}
\varepsilon_{0}(r)=2 m^{2} \tilde{\mathcal{E}}_{0}(r), \quad \varepsilon_{2}(r)=-r \frac{d}{d r} \frac{1}{r} \frac{d}{d r} \tilde{\mathcal{E}}_{2}(r), \\
\text { with } \tilde{\mathcal{E}}_{0,2}(r)=\int \frac{d^{3} \Delta}{2 E(2 \pi)^{3}} e^{-i \vec{\Delta} \cdot \vec{r}} \mathcal{E}_{0,2}(t)
\end{array}
$$

TABLE II. The free theory values of the total EMT FFs.

\begin{tabular}{lcccccc}
\hline \hline EMT FFs & $\mathcal{E}_{0}(t)$ & $\mathcal{E}_{2}(t)$ & $\mathcal{J}(t)$ & $\mathcal{D}_{0}(t)$ & $\mathcal{D}_{2}(t)$ & $\mathcal{D}_{3}(t)$ \\
\hline Free theory & 1 & 0 & 1 & $\frac{1}{3}-4 h$ & -1 & 0 \\
\hline \hline
\end{tabular}

[the definition of Eq. (23) is used for other FFs in the following], and the irreducible (symmetric and traceless) tensor of $n$th rank is [14]

$Y_{n}^{i_{1} i_{2} \cdots i_{n}}=\frac{(-1)^{n}}{(2 n-1) ! !} r^{n+1} \partial^{i_{1}} \cdots \partial^{i_{n}} \frac{1}{r}$

i.e., $Y_{0}=1, \quad Y_{1}^{i}=\frac{r^{i}}{r}, \quad Y_{2}^{i k}=\frac{r^{i} r^{k}}{r^{2}}-\frac{1}{3} \delta^{i k}$, etc.

Note there are obvious relations $\delta^{i_{l} i_{m}} Y_{n}^{i_{1} i_{2} \cdots i_{n}}=0$ and $\int d \Omega Y_{2}^{i k}=0$.

In Ref. [14], more general tensor quantities are introduced for a particle of arbitrary spin,

$$
M_{n}^{k_{1} \cdots k_{n}}=\int d^{3} \mathbf{r} r^{n} Y_{n}^{k_{1} \cdots k_{n}} T^{00}(\vec{r}),
$$

which corresponds to $2^{n}$ multipoles of the energy distribution. Here $T^{00}(\vec{r})=T^{00}(\vec{r}, \sigma, \sigma)$. Note that only even $n$ are allowed by the $P$-parity conservation. Obviously,

$$
M_{0}=m A_{0}(0)=m
$$

which gives the normalization

$$
A_{0}(0)=\sum_{a} A_{0}^{a}(t)=1 .
$$

The function $\varepsilon_{2}(r)$ gives the quadrupole distribution of the energy inside the particles and describes the deviation of the hadron's shape from the spherical one for the hadrons with spin larger than $1 / 2$. Obviously, it satisfies the condition $\int d^{3} r \varepsilon_{2}(r)=0$. For a free spin one particle one obtains (see Table II) that the quadrupole energy distribution is zero, intuitively a clear result.

\section{B. $T^{0 j}$ : Spin distribution}

The $0 k$ components of the EMT are related to the spatial distribution of the spin. The angular momentum operator in QCD is defined according to the generators of the Lorentz transformation [15],

$$
J^{i}=\frac{1}{2} \epsilon^{i j k} \int d^{3} x M^{0 j k}
$$

where $M^{0 i j}$ is the angular momentum density, expressible in terms of the energy-momentum tensor $T^{\mu \nu}$ through 


$$
M^{\alpha \mu \nu}=T^{\alpha \nu} x^{\mu}-T^{\alpha \mu} x^{\nu}
$$

From Eq. (16b), one gets

$$
T^{0 j}\left(\vec{r}, \sigma^{\prime}, \sigma\right)=\int \frac{d^{3} \Delta}{2 E(2 \pi)^{3}} e^{-i \vec{\Delta} \cdot \vec{r}}\left\langle p^{\prime}, \sigma^{\prime}\left|\hat{T}_{a}^{0 j}(0)\right| p, \sigma\right\rangle .
$$

According to Eq. (28), define the individual contributions of quarks and gluons to the particle spin $(J=1)$ as $[2,15,16]$

$$
J_{a}^{i}\left(\vec{r}, \sigma^{\prime}, \sigma\right)=\epsilon^{i j k} r^{j} T^{0 k}\left(\vec{r}, \sigma^{\prime}, \sigma\right)
$$

Inserting expression (30) [with Eq. (16b)] into Eq. (31) yields

$$
\begin{aligned}
J_{a}^{i}\left(\vec{r}, \sigma^{\prime}, \sigma\right)= & \hat{S}_{\sigma^{\prime} \sigma}^{j} \int \frac{d^{3} \Delta}{(2 \pi)^{3}} e^{-i \vec{\Delta} \cdot \vec{r}}\left[\left(\overline{\mathcal{J}}^{a}(t)+\frac{2}{3} t \frac{d \overline{\mathcal{J}}^{a}(t)}{d t}\right) \delta^{i j}\right. \\
& \left.+\left(\Delta^{i} \Delta^{j}-\frac{1}{3} \vec{\Delta}^{2} \delta^{i j}\right) \frac{d \overline{\mathcal{J}}^{a}(t)}{d t}\right]
\end{aligned}
$$

with $\overline{\mathcal{J}}^{a}(t)=\frac{m}{E} \mathcal{J}^{a}(t)$. The equation above has a form very similar to that for a spin-1/2 particle $[2,16]$. Note that the form factor $\mathcal{J}^{a}(t)$ contains the EMT nonconserving form factor $\bar{f}^{a}(t)$. In the case of spin- 0 and spin-1/2 the nonconserving form factors do not enter the spatial spin distribution.

Summing over quarks and gluons and integrating over the space yields

$$
\sum_{a} \int d^{3} r J_{a}^{i}\left(\vec{r}, \sigma^{\prime}, \sigma\right)=\hat{S}_{\sigma^{\prime} \sigma}^{i} J(0)=\hat{S}_{\sigma^{\prime} \sigma}^{i},
$$

where the individual contributions $J^{a}(0)$ add up to $\sum_{a} J^{a}(0)=J(0)$ which satisfies the normalization condition $J(0)=J=1$. Obviously, the free theory value $J^{\text {free theory }}(t)=1$ in Table I satisfies this relation.

\section{C. $T^{i j}$ stress tensor}

In the spirit of Ref. [14], the stress tensor defined by the $i j$ components of EMT in Eq. (16c) can be written generically to the quadrupole order as

$$
\begin{aligned}
T^{i j}\left(\vec{r}, \sigma^{\prime}, \sigma\right)= & \int \frac{d^{3} \Delta}{2 E(2 \pi)^{3}} e^{i \vec{\Delta} \cdot \vec{r}}\left\langle p^{\prime}, \sigma^{\prime}\left|\hat{T}_{a}^{i j}(0)\right| p, \sigma\right\rangle \\
= & p_{0}(r) \delta^{i j} \delta_{\sigma^{\prime} \sigma}+s_{0}(r) Y_{2}^{i j} \delta_{\sigma^{\prime} \sigma}+p_{2}(r) \hat{Q}^{i j} \\
& +2 s_{2}(r)\left[\hat{Q}^{i p} Y_{2}^{p j}+\hat{Q}^{j p} Y_{2}^{p i}-\delta^{i j} \hat{Q}^{p q} Y_{2}^{p q}\right] \\
& -\frac{1}{m^{2}} \hat{Q}^{k l} \partial_{k} \partial_{l}\left[p_{3}(r) \delta^{i j}+s_{3}(r) Y_{2}^{i j}\right]
\end{aligned}
$$

where the (quadrupole) pressure and shear forces function, $p_{0}(r)=\frac{1}{3} \partial^{2} \tilde{\mathcal{D}}_{0}(r), \quad s_{0}(r)=-\frac{1}{2} r \frac{d}{d r} \frac{1}{r} \frac{d}{d r} \tilde{\mathcal{D}}_{0}(r)$

$p_{2}(r)=\frac{1}{3} \partial^{2} \tilde{\mathcal{D}}_{2}(r), \quad s_{2}(r)=-\frac{1}{2} r \frac{d}{d r} \frac{1}{r} \frac{d}{d r} \tilde{\mathcal{D}}_{2}(r)$,

$p_{3}(r)=\frac{1}{3} \partial^{2} \tilde{\mathcal{D}}_{3}(r), \quad s_{3}(r)=-\frac{1}{2} r \frac{d}{d r} \frac{1}{r} \frac{d}{d r} \tilde{\mathcal{D}}_{3}(r)$,

where $\partial^{2}=\frac{1}{r^{2}} \frac{d}{d r} r^{2} \frac{d}{d r}$ is the radial part of the threedimensional (3D) Laplace operator.

Comparing with Ref. [14], we get two additional terms of quadrupole order $n=2$, which are $p_{3}(r)$ and $s_{3}(r)$ terms. The EMT conservation, $\partial_{\mu} \hat{T}^{\mu \nu}(x)=0$, implies the equilibrium equation for the static stress tensor

$$
\partial_{i} T^{i j}\left(\vec{r}, \sigma^{\prime}, \sigma\right)=0
$$

For each of the first two quadrupole orders, it is easy to check that Eqs. (35a)-(35c) satisfy the differential equations

$\frac{2}{3} s_{n}^{\prime}(r)+2 \frac{s_{n}(r)}{r}+p_{n}^{\prime}(r)=0, \quad$ with $\quad n=0,2,3$,

which guarantee the general stability condition of Eq. (36). Another three obvious relations,

$\int d^{3} \mathbf{r} p_{n}(r)=\frac{1}{3} \int d^{3} \mathbf{r} \partial^{2} \mathcal{D}_{n}(r)=0$, with $n=0,2,3$,

which shows how the internal forces balance inside a composed particle. It is a consequence of the EMT conservation, known as the von Laue condition [2,3]. We also note that the multipole pressure and shear forces distributions $\left[p_{n}(r), s_{n}(r)\right]$ satisfy the same stability equation (37) as the distributions in the spherically symmetric case of spin- 0 and spin- $1 / 2$ particles. Therefore all stability relations discussed in [2,3] are valid also for the nonspherical case of particles with higher spins.

\section{EMT-nonconserving terms}

The EMT-nonconserving terms in Eq. (4) violate the EMT conservation $\partial^{\mu} \hat{T}_{\mu \nu}(x)=0$ as

$$
\begin{aligned}
\left\langle p^{\prime},\right. & \left.\sigma^{\prime}\left|\partial^{\mu} \hat{T}_{\mu \nu}^{a}(x)\right| p, \sigma\right\rangle \\
= & i \Delta^{\mu}\left\langle p^{\prime}, \sigma^{\prime}\left|\hat{T}_{\mu \nu}^{a}(x)\right| p, \sigma\right\rangle \\
= & i e^{i \Delta x}\left[\left(\epsilon \cdot \Delta \epsilon_{\nu}^{*}+\epsilon^{\prime *} \cdot \Delta \epsilon_{\nu}-\frac{\epsilon^{\prime *} \cdot \epsilon}{2} \Delta_{\nu}\right) m^{2} \bar{f}^{a}(t)\right. \\
& \left.+\Delta_{\nu}\left(\epsilon^{\prime *} \cdot \epsilon m^{2} \bar{c}_{0}^{a}(t)+\epsilon^{\prime *} \cdot P \epsilon \cdot P \bar{c}_{1}^{a}(t)\right)\right]
\end{aligned}
$$

In the Breit frame, the 0 component of Eq. (39) is 


$$
\left\langle p^{\prime}, \sigma^{\prime}\left|\partial^{\mu} \hat{T}_{\mu 0}^{a}(x)\right| p, \sigma\right\rangle=i e^{i \Delta x}\left(\epsilon \cdot \Delta \epsilon_{0}^{\prime *}+\epsilon^{\prime *} \cdot \Delta \epsilon_{0}\right) m^{2} \bar{f}^{a}(t)=0,
$$

and the $j$ component of Eq. (39) is

$$
\begin{aligned}
\left\langle p^{\prime}, \sigma^{\prime}\left|\partial^{\mu} \hat{T}_{\mu j}^{a}(x)\right| p, \sigma\right\rangle= & i e^{i \Delta x}\left[\left(\epsilon \cdot \Delta \epsilon_{i}^{\prime *}+\epsilon^{\prime *} \cdot \Delta \epsilon_{i}-\frac{\epsilon^{\prime *} \cdot \epsilon}{2} \Delta_{i}\right) m^{2} \bar{f}^{a}(t)+\Delta_{i}\left(\epsilon^{\prime *} \cdot \epsilon m^{2} \bar{c}_{0}^{a}(t)+\epsilon^{\prime *} \cdot P \epsilon \cdot P \bar{c}_{1}^{a}(t)\right)\right] \\
= & i e^{i \Delta x}\left\{\Delta^{j} \delta_{\sigma^{\prime} \sigma^{\prime}} m^{2}\left[\frac{\bar{f}^{a}(t)}{6}-\bar{c}_{0}^{a}(t)+\frac{t}{12 m^{2}}\left[-\bar{f}^{a}(t)+2 \bar{c}_{0}^{a}(t)+\bar{c}_{1}^{a}(t)\right]-\frac{t^{2}}{48 m^{4}} \bar{c}_{1}^{a}(t)\right]\right. \\
& \left.-2 m E \Delta^{i} \hat{Q}^{i j} \bar{f}^{a}(t)+\Delta^{j} \Delta^{k} \Delta^{i} \hat{Q}^{k i}\left[\frac{1}{2} \bar{c}_{0}^{a}(t)+\frac{1}{4} \bar{c}_{1}^{a}(t)+\frac{t}{16(m+E)^{2}} \bar{f}^{a}(t)-\frac{t}{16 m^{2}} \bar{c}_{1}^{a}(t)\right]\right\} .
\end{aligned}
$$

The stability equation for the quark part of the stress tensor has the form

$$
\frac{\partial T_{i k}^{q}(\mathbf{r})}{\partial r^{k}}+f^{i}(\mathbf{r})=0
$$

This equation can be interpreted (see, e.g., the discussion in [17]) as the equilibrium equation for quark internal stress and external force (per unit of the volume) $f^{i}(\mathbf{r})$ acting on the quark subsystem from the side of the gluons. From Eq. (41) one sees that the corresponding force depends on the polarization of the spin one particle through the quadrupole spin operators $\hat{Q}^{i j}$.

\section{SUM RULES: GPD AND GFF}

By considering the Mellin moments of the vector GPDs [18], the sum rules between the GPDs and EMT FFs are found in Refs. [5,6]. The sum rules in Ref. [5] contain only conserving EMT FFs (six out of a total of nine FFs). In a recent paper [9], the polynomiality sum rules for all leading-twist quark and gluon generalized parton distributions of spin one targets are given. The generalized form factors in the polynomiality condition for GPDs of spin one particles in [9] are connected to the gravitational form factors as shown in Table I.

The quark and gluon vector GPDs are introduced in Ref. [18] for deuteron as

$$
\begin{gathered}
\left.\frac{1}{2} \int \frac{d z^{-}}{2 \pi} e^{i x P^{+} z^{-}}\left\langle p^{\prime}, \sigma^{\prime}\left|\bar{\psi}_{q}\left(-\frac{1}{2} z\right) \gamma^{+} \psi_{q}\left(\frac{1}{2} z\right)\right| p, \sigma\right\rangle\right|_{z^{+}=0, \mathbf{z}_{\perp}=0} \\
=-\left(\epsilon^{\prime *} \cdot \epsilon\right) H_{1}^{q}+\frac{\epsilon^{+}\left(\epsilon^{\prime *} \cdot P\right)+\epsilon^{\prime *+}(\epsilon \cdot P)}{P^{+}} H_{2}^{q}-\frac{2(\epsilon \cdot P)\left(\epsilon^{* *} \cdot P\right)}{M^{2}} H_{3}^{q} \\
\quad+\frac{\epsilon^{+}\left(\epsilon^{\prime *} \cdot P\right)-\epsilon^{\prime *+}(\epsilon \cdot P)}{P^{+}} H_{4}^{q}+\left\{M^{2} \frac{\epsilon^{+} \epsilon^{\prime *+}}{\left(P^{+}\right)^{2}}+\frac{1}{3}\left(\epsilon^{\prime *} \cdot \epsilon\right)\right\} H_{5}^{q}, \\
\left.\left.\frac{1}{P^{+}} \int \frac{d z^{-}}{2 \pi} e^{i x P^{+} z^{-}}\left\langle p^{\prime}, \sigma^{\prime}\left|F^{b,+\eta}\left(-\frac{1}{2} z\right) F^{b,}+\left(\frac{1}{2} z\right)\right| p, \sigma\right\rangle\right|_{z^{+}=0, \mathbf{z}_{\perp}=0}\right) \\
=-\left(\epsilon^{\prime *} \cdot \epsilon\right) H_{1}^{g}+\frac{\epsilon^{+}\left(\epsilon^{\prime *} \cdot P\right)+\epsilon^{\prime *+}(\epsilon \cdot P)}{P^{+}} H_{2}^{g}-\frac{2(\epsilon \cdot P)\left(\epsilon^{\prime *} \cdot P\right)}{M^{2}} H_{3}^{g} \\
+\frac{\epsilon^{+}\left(\epsilon^{\prime *} \cdot P\right)-\epsilon^{*+}(\epsilon \cdot P)}{P^{+}} H_{4}^{g}+\left\{M^{2} \frac{\epsilon^{+} \epsilon^{\prime *+}}{\left(P^{+}\right)^{2}}+\frac{1}{3}\left(\epsilon^{\prime *} \cdot \epsilon\right)\right\} H_{5}^{g},
\end{gathered}
$$

where $q=u, d, s, \ldots$ Integrating over $x$ of Eq. (43a), one gets the conventional form factor decomposition of the vector current for a spin one particle,

$$
\left\langle p^{\prime}, \sigma^{\prime}\left|\bar{\psi}_{q}(0) \gamma^{\mu} \psi_{q}(0)\right| p, \sigma\right\rangle=-2\left(\epsilon^{*} \cdot \epsilon G_{1}^{q}(t)+2 G_{3}^{q}(t) \frac{\epsilon^{\prime *} \cdot P \epsilon \cdot P}{m^{2}}\right) P^{\mu}+2 G_{2}^{q}(t)\left(\epsilon^{\mu} \epsilon^{\prime *} \cdot P+\epsilon^{\prime * \mu} \epsilon \cdot P\right) .
$$

So, for the quark GPDs, one has [18]

$$
\begin{aligned}
& \int_{-1}^{1} d x H_{i}^{q}(x, \xi, t)=G_{i}^{q}(t) \quad(i=1,2,3), \\
& \int_{-1}^{1} d x H_{i}^{q}(x, \xi, t)=0 \quad(i=4,5) .
\end{aligned}
$$


The charge, magnetic, and quadrupole form factors can be expressed in terms of $G_{i}=\sum_{q} G_{i}^{q}$ as $\left(\eta=-t / 4 m^{2}\right)$

$$
\begin{aligned}
& G_{C}(t)=G_{1}(t)+\frac{2}{3} \eta G_{Q}(t) \\
& G_{M}(t)=G_{2}(t) \\
& G_{Q}(t)=G_{1}(t)-G_{2}(t)+(1+\eta) G_{3}(t),
\end{aligned}
$$

normalized by the charge $G_{C}(0)=1$, magnetic moment $G_{M}(0)=\mu_{S=1} /(2 m)$, and quadrupole moment $G_{Q}(0)=Q_{S=1} / m^{2}$.

The ++ components of Eq. (2) are

$$
\begin{aligned}
T_{q}^{++}(x) & =\frac{1}{2} \bar{\psi}_{q}\left(-i \overleftarrow{\mathcal{D}}^{+} \gamma^{+}+i \overrightarrow{\mathcal{D}}^{+} \gamma^{+}\right) \psi_{q}(x), \\
T_{g}^{++}(x) & =F^{b,+\eta} F^{b,}{ }_{\eta}^{+}(x),
\end{aligned}
$$

or

$$
\begin{aligned}
T_{q}^{++}(0)= & \left(P^{+}\right)^{2} \int x d x \int \frac{d z^{-}}{2 \pi} e^{i x P^{+} z^{-}}\left[\bar{\psi}_{q}\left(-\frac{1}{2} z\right) \gamma^{+} \psi_{q}\left(\frac{1}{2} z\right)\right]_{z^{+}=0, \mathbf{z}_{\perp}=0} \\
= & {\left[2\left(P^{+}\right)^{2}\left(-\epsilon^{\prime *} \cdot \epsilon A_{0}^{q}(t)+\frac{\epsilon^{\prime *} \cdot P \epsilon \cdot P}{m^{2}} A_{1}^{q}(t)\right)+\frac{1}{2}\left(\Delta^{+}\right)^{2}\left(\epsilon^{\prime *} \cdot \epsilon D_{0}^{q}(t)+\frac{\epsilon^{\prime *} \cdot P \epsilon \cdot P}{m^{2}} D_{1}^{q}(t)\right)\right.} \\
& +4 P^{+}\left(\epsilon^{\prime *+} \epsilon \cdot P+\epsilon^{+} \epsilon^{\prime *} \cdot P\right) J^{q}(t)+\left[\epsilon^{+} \epsilon^{\prime *+} \Delta^{2}-2 \epsilon^{\prime *+} \Delta^{+} \epsilon \cdot P+2 \epsilon^{+} \Delta^{+} \epsilon^{\prime *} \cdot P\right] E^{q}(t) \\
& +2 \epsilon^{+} \epsilon^{\prime *+} m^{2} \bar{f}^{q}(t), \\
T_{g}^{++}(0)= & P^{+} \int d x \int \frac{d z^{-}}{2 \pi} e^{i x P^{+} z^{-}}\left[F^{b,+\eta}\left(-\frac{1}{2} z\right) F^{b,}{ }_{\eta}^{+}\left(\frac{1}{2} z\right)\right]_{z^{+}=0, \mathbf{z}_{\perp}=0},
\end{aligned}
$$

where $T_{g}^{++}(0)$ is similar with $T_{q}^{++}(0)$. Compare Eqs. (48) with (43), and we get the polynomiality property of vector GPDs as

$\int_{-1}^{1} x d x H_{1}^{q}(x, \xi, t)=A_{0}^{q}(t)-\xi^{2} D_{0}^{q}(t)+\frac{t}{6 m^{2}} E^{q}(t)+\frac{1}{3} \bar{f}^{q}(t)$,

$\int_{-1}^{1} x d x H_{2}^{q}(x, \xi, t)=2 J^{q}(t)$

$\int_{-1}^{1} x d x H_{3}^{q}(x, \xi, t)=-\frac{1}{2}\left[A_{1}^{q}(t)+\xi^{2} D_{1}^{q}(t)\right]$,

$\int_{-1}^{1} x d x H_{4}^{q}(x, \xi, t)=-2 \xi E^{q}(t)$,

$\int_{-1}^{1} x d x H_{5}^{q}(x, \xi, t)=\frac{t}{2 m^{2}} E^{q}(t)+\bar{f}^{q}(t)$,

for the quark parts and

$$
\begin{aligned}
& \int_{-1}^{1} d x H_{1}^{g}(x, \xi, t)=A_{0}^{g}(t)-\xi^{2} D_{0}^{g}(t)+\frac{t}{6 m^{2}} E^{q}(t)+\frac{1}{3} \bar{f}^{g}(t), \\
& \int_{-1}^{1} d x H_{2}^{g}(x, \xi, t)=2 J^{g}(t),
\end{aligned}
$$

$$
\int_{-1}^{1} d x H_{3}^{g}(x, \xi, t)=-\frac{1}{2}\left[A_{1}^{g}(t)+\xi^{2} D_{1}^{g}(t)\right],
$$

$\int_{-1}^{1} d x H_{4}^{g}(x, \xi, t)=-2 \xi E^{g}(t)$,

$\int_{-1}^{1} d x H_{5}^{g}(x, \xi, t)=\frac{t}{2 m^{2}} E^{g}(t)+\bar{f}^{g}(t)$,

for the gluon part. Equations (49b) and (50b) give the spin one version of the $\mathrm{X}$. Ji sum rule $[5,11,15]$. Note that in $H_{1,5}^{q}\left(H_{1,5}^{g}\right)$ it contains the EMT-nonconserving GFFs $\bar{f}^{q}$ $\left(\bar{f}^{g}\right)$. Thus it is useful to rewrite them as

$$
\begin{aligned}
\int_{-1}^{1} x d x\left[H_{1}^{q}(x, 0, t)-\frac{1}{3} H_{5}^{q}(x, 0, t)\right] & =A_{0}^{q}(t), \\
\int_{-1}^{1} d x\left[H_{1}^{g}(x, 0, t)-\frac{1}{3} H_{5}^{g}(x, 0, t)\right] & =A_{0}^{g}(t) .
\end{aligned}
$$

In the GPD approach, the normalization for $A_{0}$ in Eq. (27) is the energy-momentum sum rule,

$$
\begin{aligned}
& \int_{-1}^{1} d x\left[\sum_{q} x H_{1}^{q}(x, 0,0)+H_{1}^{g}(x, 0,0)\right. \\
& \left.-\frac{1}{3}\left(\sum_{q} x H_{5}^{q}(x, 0,0)+H_{5}^{g}(x, 0,0)\right)\right]=1,
\end{aligned}
$$


and the normalization for $J$ in Eq. (33) gives the sum rule,

$$
\frac{1}{2} \int_{-1}^{1} d x\left[\sum_{q} x H_{2}^{q}(x, 0,0)+H_{2}^{g}(x, 0,0)\right]=1 .
$$

As discussed in Ref. [18], the forward limit $(\xi=t=0)$ of GPDs $H_{1}$ and $H_{5}$ can give the parton distribution functions (PDFs), and therefore $H_{1,5}$ are, respectively, related to the deep inelastic scattering structure functions $F_{1}(x)$ and $b_{1}(x)$, which are worked out by Hoodbhoy et al. [10] for spin one targets. Thus, in terms of PDFs, the normalization for $A_{0}$ in Eq. (52) corresponds to the energymomentum conservation sum rule of PDFs, and, in the forward limit, the sum rule for $H_{5}$ in Eq. (45) recovers the parton model sum rule $\int b_{1}(x) d x=0$ [19].

\section{SUMMARY}

In this paper, we formulate the EMT form factors for spin one hadrons. The energy density, spin distribution, and stress tensor are given. The pressure and shear forces functions are found in terms of multipole expansion as the spin one particle is not spherically symmetric. The sum rules between the GFFs and GPDs are derived.

\section{ACKNOWLEDGMENTS}

We are grateful to Cedric Lorce, Kirill Semenov-TianShansky, and Peter Schweitzer for illuminating discussions and inspiration. This work was supported by CRC110 (DFG) and the State Scholarship Fund No. 201804910428 (CSC) and the DAAD Research Grant No. 57381332.

Note added.-While finishing the present manuscript we became aware of the recent Ref. [11] where the EMT form factors for spin one particles were also considered.

\section{APPENDIX: BREIT FRAME FORMULAS}

The matrix elements of the spin one quadrupole operator in Eq. (11),

$$
\begin{aligned}
\hat{Q}_{\sigma^{\prime} \sigma}^{i k} & =\frac{1}{2}\left(\hat{S}^{i} \hat{S}^{k}+\hat{S}^{k} \hat{S}^{i}-\frac{4}{3} \delta^{i k}\right)_{\sigma^{\prime} \sigma} \\
& =\frac{1}{3} \delta_{i j} \delta_{\sigma^{\prime} \sigma}-\frac{1}{2}\left(\hat{\epsilon}_{\sigma^{\prime} j}^{*} \hat{\epsilon}_{\sigma i}+\hat{\epsilon}_{\sigma^{\prime} i}^{*} \hat{\epsilon}_{\sigma j}\right)
\end{aligned}
$$

where $\sigma=\{+,-, 0\}$, and

$$
\hat{S}^{i} \hat{S}^{j}-\hat{S}^{j} \hat{S}^{i}=i \epsilon^{i j k} \hat{S}^{k}
$$

In the Breit frame, the initial (final) momentum $p^{\mu}\left(p^{\prime \mu}\right)$ has the relation $P^{\mu}=\left(p^{\mu}+p^{\prime \mu}\right) / 2=(E, 0,0,0)$ and
$\Delta^{\mu}=p^{\prime \mu}-p^{\mu}=(0, \vec{\Delta})$. So $\vec{p}=-\vec{p}^{\prime}=-\vec{\Delta} / 2$ and $p^{0}=$ $p^{\prime 0}=E=\sqrt{m^{2}-t / 4}$ with $t=\Delta^{2}$. So initial and final polarization vectors are

$$
\begin{gathered}
\epsilon^{\mu}(p, \sigma)=\left(-\frac{\vec{\Delta} \cdot \hat{\epsilon}_{\sigma}}{2 m}, \hat{\epsilon}_{\sigma}+\frac{\vec{\Delta} \cdot \hat{\epsilon}_{\sigma}}{4 m(m+E)} \vec{\Delta}\right), \\
\epsilon^{\mu}\left(p^{\prime}, \sigma^{\prime}\right)=\left(\frac{\vec{\Delta} \cdot \hat{\epsilon}_{\sigma^{\prime}}}{2 m}, \hat{\epsilon}_{\sigma^{\prime}}+\frac{\vec{\Delta} \cdot \hat{\epsilon}_{\sigma^{\prime}}}{4 m(m+E)} \vec{\Delta}\right) .
\end{gathered}
$$

So one can get the following useful relations [here $\hat{Q}_{k l}=$ $\hat{Q}_{\sigma^{\prime} \sigma}^{k l}$ is the matrix element and $\epsilon^{\prime * \mu}=\epsilon_{\sigma^{\prime}}^{* \mu}=\epsilon^{* \mu}\left(p^{\prime}, \sigma^{\prime}\right)$, $\epsilon^{\mu}=\epsilon_{\sigma}^{\mu}=\epsilon^{\mu}(p, \sigma)$; note $\left.t=\Delta^{2}\right]$ :

$$
\begin{aligned}
\left(\hat{\epsilon}_{\sigma^{\prime}}^{*} \cdot \vec{\Delta}\right)\left(\hat{\epsilon}_{\sigma^{\prime}} \cdot \vec{\Delta}\right) & =-\frac{t}{3} \delta_{\sigma^{\prime} \sigma}-\hat{Q}_{k l} \Delta_{k} \Delta_{l}, \\
\epsilon_{\sigma^{\prime}}^{*} \cdot \epsilon_{\sigma} & =\left(\frac{t}{6 m^{2}}-1\right) \delta_{\sigma^{\prime} \sigma}+\frac{1}{2 m^{2}} \hat{Q}_{k l} \Delta_{k} \Delta_{l}, \\
\epsilon_{\sigma^{\prime}, 0}^{*} \epsilon_{\sigma^{\prime}, 0} & =\frac{t}{12 m^{2}} \delta_{\sigma^{\prime} \sigma}+\frac{1}{4 m^{2}} \hat{Q}_{k l} \Delta_{k} \Delta_{l}, \\
\epsilon_{\sigma} \cdot \Delta & =-\frac{E}{m} \hat{\epsilon}_{\sigma} \cdot \vec{\Delta}, \\
\epsilon_{\sigma^{\prime}}^{*} \cdot \Delta & =-\frac{E}{m} \hat{\epsilon}_{\sigma^{\prime}}^{*} \cdot \vec{\Delta}, \\
\left(\epsilon_{\sigma^{\prime}}^{*} \cdot \Delta\right)\left(\epsilon_{\sigma} \cdot \Delta\right) & =-\frac{E^{2}}{m^{2}}\left(\frac{t}{3} \delta_{\sigma^{\prime} \sigma}+\hat{Q}_{k l} \Delta^{k} \Delta^{l}\right), \\
\epsilon_{\sigma^{\prime}, 0}^{*} \epsilon_{\sigma} \cdot \Delta & =\frac{E}{2 m^{2}}\left(\frac{t}{3} \delta_{\sigma^{\prime} \sigma}+\hat{Q}_{k l} \Delta^{k} \Delta^{l}\right), \\
\epsilon_{\sigma, 0} \epsilon_{\sigma^{\prime}}^{*} \cdot \Delta & =-\epsilon_{\sigma^{\prime}, 0}^{*} \epsilon_{\sigma} \cdot \Delta,
\end{aligned}
$$

$$
\begin{aligned}
& \epsilon_{\sigma^{\prime}, j}^{*} \epsilon_{\sigma} \cdot \Delta-\epsilon_{\sigma, j} \epsilon_{\sigma^{\prime}}^{*} \cdot \Delta=\frac{i E}{m} \Delta_{k} \epsilon^{k j l} \hat{S}_{\sigma^{\prime} \sigma}^{l}, \\
& \epsilon_{\sigma, 0} \epsilon_{\sigma^{\prime}, j}^{*}+\epsilon_{\sigma^{\prime}, 0}^{*} \epsilon_{\sigma, j}=\frac{i}{2 m} \Delta_{k} \epsilon^{k j l} \hat{S}_{\sigma^{\prime} \sigma}^{l}, \\
& \epsilon_{\sigma, i} \epsilon_{\sigma^{\prime}, j}^{*}+\epsilon_{\sigma^{\prime}, i}^{*} \epsilon_{\sigma^{\prime} j}=\left(\frac{2}{3} \delta_{i j}+\frac{1}{6 m^{2}} \Delta_{i} \Delta_{j}\right) \delta_{\sigma^{\prime} \sigma}-2 \hat{Q}_{i j}
\end{aligned}
$$

$$
\begin{aligned}
& -\frac{1}{2 m(m+E)}\left(\Delta_{i} \Delta_{k} \hat{Q}_{k j}+\Delta_{j} \Delta_{k} \hat{Q}_{k i}\right) \\
& -\frac{1}{8 m^{2}(m+E)^{2}} \Delta_{i} \Delta_{j} \Delta_{k} \Delta_{l} \hat{Q}_{k l} .
\end{aligned}
$$


[1] M. V. Polyakov, Phys. Lett. B 555, 57 (2003).

[2] M. V. Polyakov and P. Schweitzer, Int. J. Mod. Phys. A 33, 1830025 (2018).

[3] C. Lorcé, H. Moutarde, and A. P. Trawiński, Eur. Phys. J. C 79, 89 (2019).

[4] B. R. Holstein, arXiv:gr-qc/0607054.

[5] Z. Abidin and C.E. Carlson, Phys. Rev. D 77, 095007 (2008).

[6] S. K. Taneja, K. Kathuria, S. Liuti, and G. R. Goldstein, Phys. Rev. D 86, 036008 (2012).

[7] A. V. Belitsky and A. V. Radyushkin, Phys. Rep. 418, 1 (2005).

[8] M. V. Polyakov, K. M. Semenov-Tian-Shansky, and B. D. Sun (to be published).

[9] W. Cosyn, A. Freese, and B. Pire, Phys. Rev. D 99, 094035 (2019).

[10] P. Hoodbhoy, R. L. Jaffe, and A. Manohar, Nucl. Phys. B312, 571 (1989).
[11] W. Cosyn, S. Cotogno, A. Freese, and C. Lorcé, Eur. Phys. J. C 79, 476 (2019).

[12] S. Weinberg, The Quantum Theory of Fields. Vol. 1: Foundations (Cambridge University Press, Cambridge, England, 2005).

[13] W. Detmold, D. Pefkou, and P. E. Shanahan, Phys. Rev. D 95, 114515 (2017).

[14] M. V. Polyakov and P. Schweitzer, arXiv:1812.06143.

[15] X. D. Ji, Phys. Rev. Lett. 78, 610 (1997).

[16] C. Lorcé, L. Mantovani, and B. Pasquini, Phys. Lett. B 776, 38 (2018).

[17] M. V. Polyakov and H. D. Son, J. High Energy Phys. 09 (2018) 156.

[18] E. R. Berger, F. Cano, M. Diehl, and B. Pire, Phys. Rev. Lett. 87, 142302 (2001).

[19] F. E. Close and S. Kumano, Phys. Rev. D 42, 2377 (1990). 